\title{
Neutron star blackbody contraction during flaring in X 1624-490
}

\author{
M. Bałucińska-Church ${ }^{1,2}$, R. Barnard ${ }^{1}$, M. J. Church ${ }^{1,2}$, and A. P. Smale ${ }^{3}$ \\ 1 School of Physics and Astronomy, University of Birmingham, Birmingham B15 2TT, UK \\ e-mail: mbc@star.sr.bham.ac.uk \\ 2 Institute of Astronomy, Jagiellonian University, ul. Orla 171, 30-244 Cracow, Poland \\ 3 Laboratory for High Energy Astrophysics, Code 662, NASA/Goddard Space Flight Center, \\ Greenbelt, MD 20771, USA \\ Also, Universities Space Research Association \\ e-mail: alan@osiris.gsfc.nasa.gov
}

Received 12 January 2001 / Accepted 16 August 2001

\begin{abstract}
We present results of an extensive investigation of the physical changes taking place in the emission regions of the LMXB X 1624-490 during strong flaring in observations made using RXTE in 1997 and 1999. Based on the detailed light curve, we propose that the flaring consists of a superposition of X-ray bursts. It is shown that major changes take place in the blackbody emission component, the temperature $k T_{\mathrm{BB}}$ increasing to $\sim 2.2 \mathrm{keV}$ in flaring. Remarkably, the blackbody area decreases by a factor of $\sim 5$ in flaring. During flare evolution, the blackbody luminosity remains approximately constant, constituting a previously unknown Eddington limiting effect which we propose is due to radiation pressure of the blackbody as $k T_{\mathrm{BB}}$ increases affecting the inner disk or accretion flow resulting in a decreased emitting area on the star. We argue that the large decrease in area cannot be explained in terms of modification of the blackbody spectrum by electron scattering in the atmosphere of the neutron star. The height of the emitting region on the non-flaring neutron star is shown to agree with the height of the inner radiatively-supported accretion disk as found for sources in the ASCA survey of LMXB of Church \& Bałucińska-Church (2001). The decrease in height during flaring is discussed in terms of possible models, including radial accretion flow onto the stellar surface and the theory of accretion flow spreading on the neutron star surface of Inogamov \& Sunyaev (1999). We demonstrate that the intensity of the broad iron line at $6.4 \mathrm{keV}$ is strongly correlated with the luminosity of the blackbody emission from the neutron star, and discuss the probable origin of this line in the ADC. Finally, possible reasons for non-detection of a reflection component in this source, and LMXB in general, are discussed.
\end{abstract}

Key words. X rays: stars - stars: individual: X 1624-490 - stars: neutron - binaries: close accretion: accretion disks

\section{Introduction}

$\mathrm{X} 1624-490$ is one of the most unusual members of the class of dipping Low Mass X-ray Binary (LMXB) sources exhibiting periodic dips in X-ray intensity. It is generally accepted that dipping is due to absorption in the bulge in the outer accretion disk where the accretion flow from the companion impacts (White \& Swank 1982). X 1624-490 has the longest orbital period of the dipping sources at $\sim 21 \mathrm{hr}$. Dipping is deep, $75 \%$ in the band $1-10 \mathrm{keV}$, and the source also exhibits strong flaring in which the X-ray flux can increase by $30 \%$ over timescales of a few thousand seconds (Church \& Bałucińska-Church 1995). Flaring has been known in LMXB for many years since it was recognized that Sco X-1 had quiescent states and active states

Send offprint requests to: M. Bałucińska-Church, e-mail: mbc@star.sr.bham.ac.uk in which strong X-ray flaring took place (Canizares et al. 1975). White et al. (1985), using the Exosat GSPC, showed that the continuum between $2-25 \mathrm{keV}$ could be modelled by a blackbody plus unsaturated Comptonization spectrum and that the flaring is caused by increases in the blackbody luminosity from $10 \%$ to $40 \%$ of the total. The blackbody temperature increased significantly in flaring together with some increase in emitting area. Hasinger et al. (1989) realized that Sco X-1 was a Z-track source, the source having different spectral states forming a Ztrack on a hardness-intensity diagram with associated changes in QPO behaviour. It was also realized that several other bright sources exhibited similar flaring to that in Sco X-1. However, since then, little work has been carried out on flaring in LMXB, physical understanding of the flaring phenomenon is limited, and the transition between 
bursting in less bright LMXB and flaring in brighter sources is not understood.

Investigation of X-ray dipping has however, been more extensive. The depth, duration and spectral evolution in dipping vary considerably from source to source. Moreover, spectral analysis of dipping sources provides information not available in non-dipping sources. For example, spectral models are more strongly constrained by having to fit non-dip and dip data, thus showing clearly the nature of the emission. It has been shown that the dipping LMXB sources are well described by assuming point-like blackbody emission from the neutron star plus Comptonized emission from an extended Accretion Disk Corona (ADC) (e.g. Church \& Bałucińska-Church 1995; Church 2001). In addition, the dip ingress and egress times can be used to obtain the sizes of the extended emission region if the absorber has larger angular extent that the source regions, as proven by dipping being $100 \%$ deep at any energy, which is often the case. This provides a radius for the ADC, $r_{\mathrm{ADC}}$, typically $50000 \mathrm{~km}$ or $15 \%$ of the accretion disk radius, although in $\mathrm{X} 1624-490, r_{\mathrm{ADC}}$ is $5.3 \times 10^{10} \mathrm{~cm}$ or $50 \%$ of the disk radius (Church 2001). There is also evidence that the ADC is "thin", having height-to-radius ratio of $\sim 10 \%$ (Smale et al. 2001). This shows that the ADC is a very extended, flat region above the accretion disk, contrary to theoretical suggestions often made that the Comptonizing region may be localized to the neighbourhood of the neutron star.

Recent results of an $A S C A$ and BeppoSAX survey of LMXB (Church \& Bałucińska-Church 2001), reveal a surprising result for the area of the neutron star emitting blackbody radiation for sources of different luminosities. When it is assumed that the emission region is an equatorial strip of half-height $h$, it is found that there is good agreement between $h$ and $H$, the half-height of the inner disk, assumed to be radiatively supported. It is not clear at the moment whether there is a direct causal link between these parameters, for example, if material flowed radially between the inner disk and the star, or whether there is an indirect link such that $H$ is a measure of the total source luminosity $L_{\text {Tot }}$ and some physical process depending on $L_{\text {Tot }}$ determines the extent of the emitting area. In particular, Inogamov \& Sunyaev (1999) have shown that accretion flow regarded as a fluid meeting the neutron star at the equator is expected to spread vertically up the star to a height dependent on $L_{\text {Tot }}$. A preliminary comparison of this model with the survey results (Church et al. 2001) shows that observed blackbody luminosities are $\sim 3$ times larger than implied by the theory, suggesting that radial flow may be the dominant factor determining the blackbody luminosity. The results of the present work will be viewed (Sects. 3 and 4 ) in terms of the $h=H$ relation.

X 1624-490 was previously observed in a long Exosat observation of $220 \mathrm{ks}$ and with Ginga (Jones \& Watson 1989). The Exosat ME data revealed an apparently stable lower level of dipping supporting the presence of two emission components, one of which was totally absorbed in deep dipping. Church \& Bałucińska-Church (1995) showed that the light curve at higher energies $(>5 \mathrm{keV})$ was dominated by flaring which can strongly modify the spectrum. Thus if flaring occurs simultaneously with dipping, the investigation of spectral evolution in dipping will be difficult. By selecting sections of non-dip and dip data without apparent flaring, the above two-component model was found to fit the data well showing that in deep dipping the blackbody component was totally absorbed, and that the Comptonized component was relatively little absorbed. However, with only one non-dip and one deep dip spectrum it was not possible to determine the extent of absorption of the Comptonized component. The Galactic column density of X 1624-490 is very high $\left(\sim 8 \times 10^{22}\right.$ atom $\left.\mathrm{cm}^{-2}\right)$ so that a dust scattered halo of the source is expected, and Angelini et al. (1997) demonstrated an excess of the surface brightness above the point spread function in $A S C A$ GIS.

The 60 ks observation with BeppoSAX of 1999, August 11 was almost free of flaring and fitting the radial intensity profile of the MECS instruments in four energy bands between 2.5 and $6.5 \mathrm{keV}$ allowed a value of the optical depth to dust scattering of $2.4 \pm 0.4$ to be determined (Bałucińska-Church et al. 2000). Moreover, the very broad band of BeppoSAX allowed the Comptonization cut-off energy to be determined for the first time, this having the value of $12_{-5}^{+14} \mathrm{keV}$. Spectral evolution in dipping could be well-described in terms of an emission model consisting of point-like blackbody emission from the neutron star plus extended Comptonized emission from the ADC. It was shown that using a disk blackbody model instead of a simple blackbody resulted in an unphysical inner radius of $0.4 \mathrm{~km}$, so this possibility could be discounted. Similar unphysical results were obtained for this model for the majority of the LMXB in the $A S C A$ survey allowing us to associate the blackbody emission with the neutron star in LMXB in general. Additionally, the extended size of the ADC and high optical depth imply that all of the thermal emission from the disk will be reprocessed in the ADC. Thus, we do not expect any contribution to the observed thermal emission from the disk.

Several $R X T E$ observations have been made by the authors including observations on 1997, January 6-7, 1997, May 4, 6, 11 which failed to capture dipping. A 4-day observation with RXTE was made in 1999, September spanning 4 orbital cycles with deep dipping and extensive flaring. Results of analysis of the spectral evolution in dipping are presented in Paper I (Smale et al. 2001). The orbital period has not been refined since the Exosat determination of $21 \pm 2 \mathrm{hr}$ (Watson et al. 1985). In Paper I, an orbital ephemeris and improved period of $20.8778 \pm 0.0003 \mathrm{hr}$ are presented based on $4.5 \mathrm{yr}$ of $R X T E$ ASM data. In addition, a search failed to detect QPO at frequencies from $\mathrm{Hz}$ to $\mathrm{kHz}$, which may be related to the high luminosity of the source. The much higher count rate and observation duration in the 1999, September observation with $R X T E$ revealed several new features including the dominance of electron scattering in the early stages of dipping. The main deep dips were surrounded by shallow 
"shoulders of dipping" in which a vertical shift of the spectrum relative to the non-dip spectrum was seen between 2.5 and $25 \mathrm{keV}$. This demonstrated that electron scattering was dominant and photoelectric absorption was not detectable indicating the highly ionized state of the outer absorber produced by the high luminosity of X 1624-490. A broad iron line at $\sim 6.4 \mathrm{keV}$ was first detected by Asai et al. (2000) using $A S C A$. Spectral evolution in dipping could be well described using the above two-component emission model having the basic form: $\mathrm{AG} *(\mathrm{AB} * \mathrm{BB}+$ PCF* $(\mathrm{CPL}+\mathrm{GAU}))$, where AG represents Galactic absorption, $\mathrm{AB}$ additional absorption of the point-like blackbody in dipping, $\mathrm{PCF}$ the progressive covering of the extended Comptonized emission (described as a cut-off power law) and GAU the Gaussian line. Good fits were obtained by including the $6.4 \mathrm{keV}$ line in the same covering term as the Comptonized emission, showing that the line probably originates in the ADC. However, to the basic form were added complicating terms for the effects of both electron scattering and dust scattering (Smale et al. 2001).

In the present paper (Paper II), we present a study of flaring in X 1624-490 based on the observations of 1997, January and 1999, September during both of which strong flaring took place. Although flaring in Sco X-1 and similar sources has been known for many years, in which clear indications of increasing blackbody temperature were found, there has been no recent observational work aimed at understanding the physical changes taking place, or the differences between bursting and flaring sources. In addition, flaring is clearly related to the flaring branch of the Z-track class of LMXB. X 1624-490 offers a unique opportunity for investigating these problems as it is both a dipping and a flaring source.

\section{Observations}

X 1624-490 was observed using RXTE (Bradt et al. 1993) from 1999, September 27 18:22 UT-October 1 13:11 UT, for a total on-source exposure of $212 \mathrm{ks}$. In addition to results from this main observation spanning 4 orbital cycles of $20.87 \mathrm{hr}$, we present results from our observation of 1997, January 6 11:14 UT-January 7 3:28 UT. The new ephemeris (Paper I) shows that this observation, intended to capture dipping, spanned phases $0.11-0.89$ where phase 0 is dip centre, exactly missing deep dipping. However, there was a period of strong flaring and both observations are of interest because of the change in source luminosity between the observations, the 1997 observation having a luminosity (1-30 keV) of $1.06 \times 10^{38} \mathrm{erg} \mathrm{s}^{-1}$, compared with $1.35 \times 10^{38} \mathrm{erg} \mathrm{s}^{-1}$ in the 1999 observation, approaching closer to the Eddington limit for a $1.4 M_{\odot}$ object. It will be seen that this difference apparently leads to significant differences in flare development (Sect. 3). For the analysis presented here of relatively slow flare development and decay, data from the PCA instrument in the Standard 2 mode were used having a time resolution of $16 \mathrm{~s}$. The PCA consists of five Xe proportional counter units (PCUs) numbered 0 to 4 , with a combined total
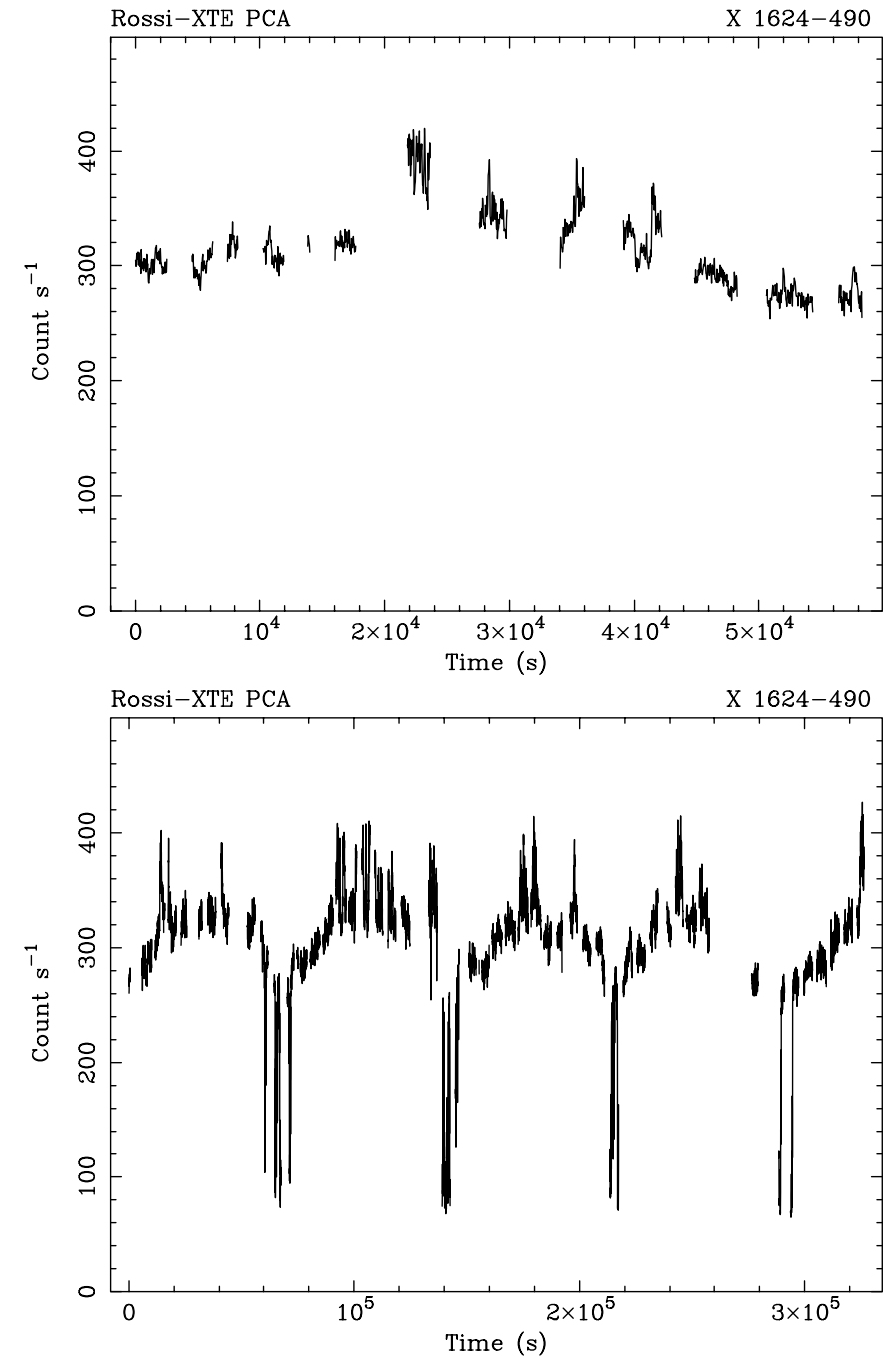

Fig. 1. PCA light curve of X 1624-490 with 32 s binning, upper curve: the 1997, January observation in the band 1.9-25 keV; lower curve: the 1999, September observation in the band 2.0$25 \mathrm{keV}$.

effective area of about $6500 \mathrm{~cm}^{2}$ (Jahoda et al. 1996). During the September 1999 observation only PCUs 0 and 2 were reliably on throughout the observation, whereas in 1997 all PCUs could be used. To allow direct comparison of the lightcurves and spectra, results are presented here for both observations using PCUs 0 and 2, the high count rate permitting this. Data were also obtained with the HEXTE phoswich detectors, which are sensitive over the energy range 15-250 keV (Rothschild et al. 1998). However, because of the relatively low break energy of the Comptonized spectrum at $12 \mathrm{keV}$ (Paper I) producing a relatively low count rate in HEXTE, and because spectral evolution during flaring takes place primarily in the band 1-20 keV, the HEXTE data provided little useful additional information.

Data analysis was carried out using the RXTE standard analysis software, FTOOLS 5.0.1. Background subtraction of the PCA data was performed using the "skyvle/skyactiv 20000131" models generated by the 


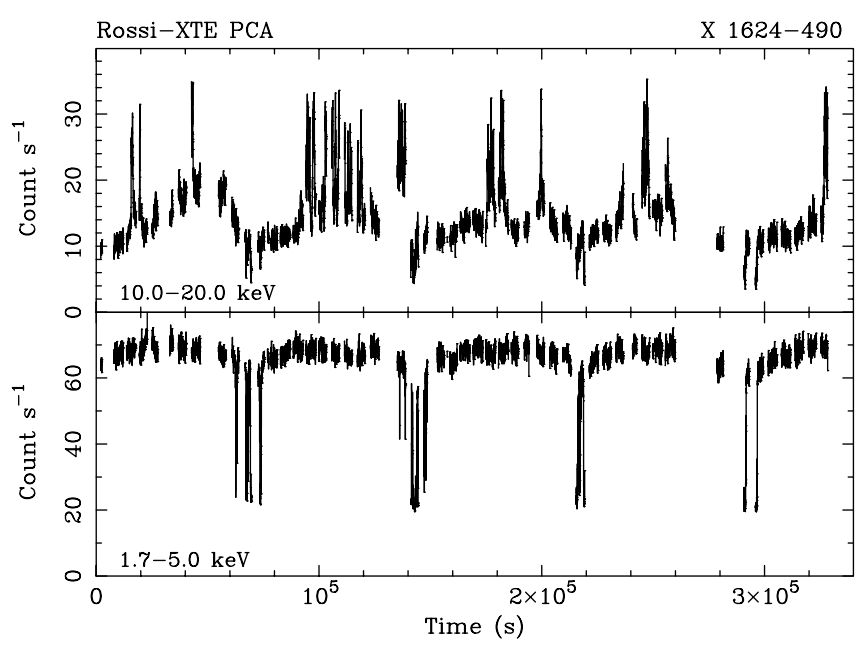

Fig. 2. PCA light curves of the 1999 observation of X 1624-490 in two energy bands: $1.7-5.0$ and 10.0-20.0 with $32 \mathrm{~s}$ binning.

RXTE PCA team. The quality of the background subtraction was checked firstly by comparing the source and background spectra and light curves at high energies $(50-100 \mathrm{keV})$ where the source no longer contributes detectable events; and also using the same models to background-subtract the data obtained during slews to and from the source. We conclude that our background subtractions in the 2-20 keV energy range are accurate to a fraction of a count per second. The quality of both the background subtraction and the response matrices used was checked by including a careful analysis of Crab calibration observations on 1999, September 26, October 13, November 8, and November 23, before and after our 1999 observation of X 1624-490 (see Paper I). Systematic errors of $1 \%$ were added to the spectra.

\section{Results}

\subsection{The X-ray lightcurves}

Figures $1 \mathrm{a}$ and $1 \mathrm{~b}$ show light curves of the two observations in the total PCA band. As the 1997 observation was made during PCA gain epoch 3, and the 1999 observation during epoch 4 , we used appropriate PCA channel ranges to give very similar energy ranges in the two light curves: 1.9-25 keV and 2.0-25 keV for 1997 and 1999, respectively. Figure 2 shows the 1999 observation in two energy bands: a low energy band (1.7-5.0 keV) in which dipping is predominant and a high energy band (10.0-20.0 keV) in which flaring dominates.

In the 1997 lightcurve, a period of strong flaring is followed almost certainly by a shoulder of dipping, i.e. the period of shallow dipping that often precedes and follows the main deep dipping. In Paper I, we showed that this consists of a reduction in intensity of the extended Comptonized emission by electron scattering in the outer layers of the absorber before the neutron star is overlapped by absorber and deep dipping commences. During the shoulder, the blackbody emission component was shown to have zero column density additional to the Galactic contribution; however, it is important to realize that this does not represent the quiescent, i.e. non-flaring, non-dipping emission which has a somewhat higher intensity. The 1999 lightcurves in Fig. 2 demonstrate vividly that while dipping is essentially a low-energy phenomenon, flaring dominates the light curves at high energies, and can only, in fact, be detected above $\sim 6 \mathrm{keV}$. The reason for this can be understood in terms of the non-flare blackbody temperature $k T_{\mathrm{BB}} \sim 1.3 \mathrm{keV}$ which increases to $\sim 2.2 \mathrm{keV}$ in flaring, so that the spectra cross over at about $6 \mathrm{keV}$, resulting in a deficit below this energy and an excess above, compared with the non-flare spectrum. Close inspection of the 1999 light curve indicates that each flare is highly structured consisting of many shorter events, and we propose here that flaring consists of a superposition of X-ray bursts occurring with much reduced separation compared with burst sources, due to the mass accretion rate being more than 10 times higher (at a luminosity of $\sim 10^{38} \mathrm{erg} \mathrm{s}^{-1}$ ).

\subsection{Spectral evolution in flaring}

\subsubsection{Selection of flare spectra}

Flare evolution is firstly displayed on X-ray hardnessintensity diagrams for both the 1997 and 1999 observations in Fig. 3 (left panels). Hardness is defined as the ratio of intensities in the bands $9.8-24.9 \mathrm{keV}$ and 4.9 $9.8 \mathrm{keV}$, and intensity is in the total band $1.9-24.9 \mathrm{keV}$. In the 1997 data (upper panel), strong flaring but only shallow dipping took place, whereas in the 1999 data (lower panel), there was both strong flaring and deep dipping. In this case, dipping begins with intensity decreases of $\sim 15 \%$ in an energy-independent way (horizontal part of Fig. 3) corresponding to a shoulder of dipping, and consistent with electron scattering of the non-dip spectrum in highly ionized outer layers of the absorber (Paper I). In deeper dipping, spectral evolution is complex, showing first a strong hardening as Comptonized emission is absorbed, then a softening in deepest dipping (at $<80 \mathrm{c} \mathrm{s}^{-1}$ ) as the blackbody emission becomes totally removed leaving the residual spectrum harder.

During flare evolution, systematic changes clearly take place in hardness-intensity very similar to the flaring branch of Z-track sources displayed on similar diagrams. Flare spectra selected for spectral analysis are also shown as average points for each spectrum in Fig. 3 (right panels). It was not initially clear that simply selecting flare spectra on the basis of intensity would be adequate to allow understanding of spectral evolution during flaring. Preliminary tests with this and other sources displaying track movement revealed that selection on intensity alone may produce scatter in the positions of the selected spectra about a line drawn through the centre of the track on the hardness-intensity plot. Such scatter leads to changes in spectral parameters not due to the development of flaring particularly in the Comptonized emission, and this complicates interpretation. Consequently it was decided 

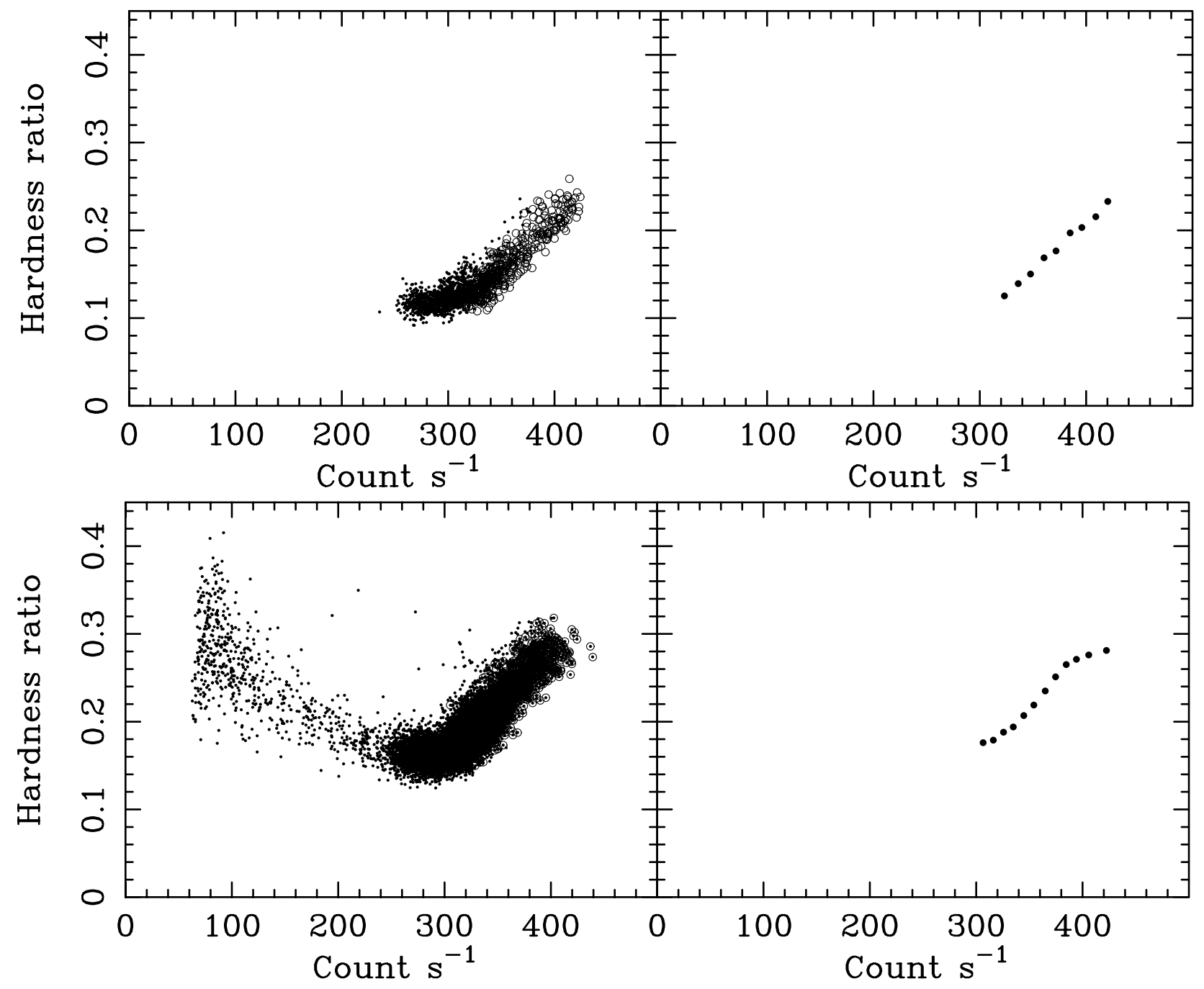

Fig. 3. Flare evolution displayed on hardness-intensity diagrams; upper panel left: the 1997 observation; lower panel left: the 1999 observation. The dots show data that was excluded during selection of flare data, i.e. data in dipping, the shoulders of dipping, or flaring during the shoulders; open circles shows data accepted for flare analysis. The right hand panels in each case show the corresponding plots for spectra selected for analysis, allowing these to be compared with the total data (see text).

to select spectra in such a way that the points lay along the centre of the track on the hardness-intensity diagram that represents flare evolution. This was done for the 1997 data by selecting data on the basis of both hardness ratio and intensity, defining essentially a box for each spectrum within Fig. 3. It can be seen that the spectra produced well represent the general track movement. In the case of the 1999 observation, spectra were first selected on the basis of intensity alone, and when these data were mapped onto the hardness-intensity diagram, it was found that these points moved systematically along the flare evolution track, obviating the need for selection on both hardness and intensity. This was a result of the much improved total count in spectra of the long observation. Comparison of results from this "track-centre" technique with initial tests showed that the behaviour during flare development becomes systematic.
For the 1997 observation, 9 flare spectra were selected using good-time intervals obtained from a hardnessintensity diagram made in the same energy bands as in Fig. 3 but using all 5 PCUs to reduce the errors on every point. Only the first 3 flares were used because the 4th flare is in the shoulder of dipping. For the 1999 observation, 12 flare spectra were selected in the same way avoiding dipping and shoulder-of-dipping data. The results of fitting spectra selected in this way are discussed in the following section. Finally, we investigated whether it is adequate to add data from different flares by the procedure of intensity selection, and analysed data from single flares including the strong flare at $\sim 180 \mathrm{ks}$ in the 1999 data (Fig. 1b). Results showed that this flare followed the same track on the hardness-intensity diagram as the total 1999 data, and spectral analysis results were also very 
Table 1. Ability of various spectral models to fit the 1999 flare spectra simultaneously. The models are variations on the twocomponent $\mathrm{BB}+\mathrm{CPL}$ model, except for the first, in which a second blackbody is added to attempt to model flaring.

\begin{tabular}{lr}
\hline Model & $\chi^{2} /$ d.o.f. \\
\hline Additional blackbody & $1038 / 589$ \\
Constant cut-off power law & $813 / 568$ \\
Constant blackbody & $1493 / 568$ \\
Constant blackbody radius & $904 / 557$ \\
Constant blackbody temperature & $1283 / 557$ \\
Constant power law index & $445 / 557$ \\
Constant power law normalization & $370 / 557$ \\
Free fitting & $354 / 546$ \\
\hline
\end{tabular}

similar. Consequently there was no need to restrict analysis to individual datasets.

\subsubsection{Spectral evolution during flaring}

In Paper I, it was shown that non-dip, non-flaring emission can be well-described by a two-component model consisting of point-like blackbody emission from the surface of the neutron star plus extended Comptonized emission from the accretion disk corona represented by a cut-off power law, plus a line at $6.4 \mathrm{keV}$. Dust scattering is significant in this source, since photons from non-dip emission are scattered into the beam in dipping due to the timedelay in scattering. As the amount lost from the beam depends on the dip intensity; there is an excess due to the difference of these terms which makes a (non-dominant) contribution to the residual count rate in deep dipping. Outside dipping, the two terms balance and no effect is expected, and the spectrum is well-modelled by the two continuum components above. The cross-section for dust scattering has a strong energy dependence $\sim E^{-2}$ therefore the limited effects that are seen are at low energies and flaring at energies above $6 \mathrm{keV}$ should not be influenced by dust scattering. In Paper I we exhaustively tested a range of one-component and two-component models using non-dip and several dip spectra, which showed that only the above model was capable of fitting all of these spectra. Consequently in the present work we use that model to investigate spectral evolution during flaring, i.e. the model $\mathrm{AG} *(\mathrm{BB}+\mathrm{CPL}+\mathrm{GAU})$ where $\mathrm{AG}$ represents Galactic absorption, BB is the neutron star blackbody, CPL is the cut-off power law representation of Comptonization in an extended ADC and GAU is the Gaussian line.

This model was firstly used to test various possibilities for flaring evolution, such as the Comptonized emission remaining constant, by fitting the 12 spectra from 1999 simultaneously, and results are shown in Table 1 . The probability of any model being correct was vanishingly small except for the last three, including attempting to model flaring by an additional blackbody component. The last model with all parameters free was more probable and acceptable at $>99 \%$ probability, and so this model was used in fitting the 1997 and 1999 observations.

Spectral fitting was carried out by simultaneously fitting all flare spectra in each of the observations using the two-component continuum model described above together with a line at $6.4 \mathrm{keV}$ with width $\sigma$ fixed at $0.4 \mathrm{keV}$ (as in Paper I) to prevent the tendency of a broad line to absorb neighbouring continuum. Initially, the Galactic column density was free, however, this is not well-determined in $R X T E$ and with free-fitting, values tended to become substantially higher $(50 \%)$ than the well-determined value from BeppoSAX of $8.6 \pm 1.0 \times 10^{22}$ atom $\mathrm{cm}^{-2}$ (Bałucińska-Church et al. 2000). Consequently, this value was fixed in the fitting. Using BeppoSAX with a spectrum extending to higher than $100 \mathrm{keV}$, it was also possible to obtain a good measurement of the Comptonization cutoff energy, while in the RXTE PCA with more restricted energy band it is difficult to get reliable values, particularly of possible changes in this parameter during flaring. We show below that the most dramatic changes in flaring take place in the blackbody component; nevertheless, we tested the effect of different approaches to the cut-off energy as follows. Firstly, we allowed this parameter to be entirely free, or free but chained between individual flare spectra in each observation. Secondly, we fixed the value for all spectra at the best-fit value obtained from BeppoSAX of $11.8 \mathrm{keV}$. Finally, we fixed the value at various values between $11.8 \mathrm{keV}$ and $100 \mathrm{keV}$. The quality of fit deteriorated as the value was increased, and maximum values of $E_{\mathrm{CO}}$ of $12 \mathrm{keV}$ (1997) and $21 \mathrm{keV}$ (1999) were found, after which $\chi^{2} /$ d.o.f. became unacceptable. With the cut-off energy free but chained between spectra, values of $\sim 5 \mathrm{keV}$ (1997) and $\sim 9 \mathrm{keV}$ (1999) were obtained. However, the results presented below were found not to be sensitive to $E_{\mathrm{CO}}$, particularly the blackbody temperature and radius, and the systematic changes in these during flare development; consequently it was decided to adopt the approach of fixing the cut-off energy at the BeppoSAX value. Spectral fitting results are shown in Table 2 for the 1997 observation and in Table 3 for the 1999 observation. In Fig. 4 we show the blackbody radius $R_{\mathrm{BB}}$, the temperature $k T_{\mathrm{BB}}$, luminosity $L_{\mathrm{BB}}$ and the power law photon index $\Gamma$ as a function of $L_{\text {Tot }}$, for the 1997 and 1999 observations, respectively.

In both observations, it was clear that strong changes take place during flaring in the blackbody component with $k T_{\mathrm{BB}}$ increasing from $\sim 1.3 \mathrm{keV}$ to more than $2 \mathrm{keV}$. Remarkably, the blackbody radius (where the emitting area is $4 \pi R_{\mathrm{BB}}^{2}$ ) decreased strongly in both observations. In the 1997 data, $L_{\mathrm{BB}}$ remained approximately constant during flaring, and similar behaviour was seen in the 1999 data for $L_{\text {Tot }}<1.5 \times 10^{38} \mathrm{erg} \mathrm{s}^{-1}$ (equal to the largest $L_{\text {Tot }}$ in 1997); at higher $L_{\text {Tot }}, L_{\mathrm{BB}}$ increased to $\sim 3.5 \times 10^{37} \mathrm{erg} \mathrm{s}^{-1}$ at the peak of flaring. Thus, it is clear that over much of flare development, the decrease of $R_{\mathrm{BB}}$ offsets the increase of $k T_{\mathrm{BB}}$ so that $L_{\mathrm{BB}}$ remains constant. A consequence of the constancy of $L_{\mathrm{BB}}$ is that the increase in total luminosity is entirely in the Comptonized 
Table 2. Spectral fitting results for the 1997 RXTE PCA spectra fitted simultaneously; $\chi^{2} /$ d.o.f. $=400 / 405.90 \%$ confidence errors are given.

\begin{tabular}{rcrccc}
\hline No. & $\begin{array}{c}k T_{\mathrm{BB}} \\
\mathrm{keV}\end{array}$ & $\begin{array}{c}R_{\mathrm{BB}} \\
\mathrm{km}\end{array}$ & $\begin{array}{c}L_{\mathrm{BB}} 10^{37} \\
\mathrm{erg} \mathrm{s}^{-1}\end{array}$ & $\Gamma$ & $\begin{array}{r}L_{\mathrm{CPL} 10^{37}} \\
\mathrm{erg} \mathrm{s}^{-1}\end{array}$ \\
\hline 1 & $1.34 \pm 0.02$ & $10.2 \pm 0.4$ & $4.3 \pm 0.2$ & $2.09 \pm 0.06$ & 7.6 \\
2 & $1.40 \pm 0.03$ & $8.9 \pm 0.4$ & $4.0 \pm 0.2$ & $2.04 \pm 0.06$ & 8.1 \\
3 & $1.45 \pm 0.03$ & $8.3 \pm 0.4$ & $4.0 \pm 0.2$ & $1.98 \pm 0.06$ & 8.3 \\
4 & $1.54 \pm 0.05$ & $7.3 \pm 0.5$ & $3.8 \pm 0.3$ & $1.90 \pm 0.07$ & 8.8 \\
5 & $1.59 \pm 0.06$ & $6.7 \pm 0.6$ & $3.8 \pm 0.3$ & $1.88 \pm 0.08$ & 9.6 \\
6 & $1.78 \pm 0.08$ & $5.4 \pm 0.5$ & $3.8 \pm 0.3$ & $1.91 \pm 0.10$ & 9.8 \\
7 & $1.80 \pm 0.07$ & $5.4 \pm 0.5$ & $3.8 \pm 0.3$ & $1.85 \pm 0.08$ & 10.0 \\
8 & $1.90 \pm 0.07$ & $4.8 \pm 0.4$ & $3.9 \pm 0.3$ & $1.78 \pm 0.07$ & 10.9 \\
9 & $1.98 \pm 0.10$ & $4.5 \pm 0.5$ & $4.1 \pm 0.4$ & $1.79 \pm 0.10$ & 11.1 \\
\hline
\end{tabular}

Table 3. Equivalent results for the 1999 data. $\chi^{2} /$ d.o.f. $=$ $354 / 546$ for 12 spectra fitted simultaneously.

\begin{tabular}{rccccc}
\hline No. & $\begin{array}{c}k T_{\mathrm{BB}} \\
\mathrm{keV}\end{array}$ & $\begin{array}{c}R_{\mathrm{BB}} \\
\mathrm{km}\end{array}$ & $\begin{array}{c}L_{\mathrm{BB}} 10^{37} \\
\mathrm{erg} \mathrm{s}^{-1}\end{array}$ & $\Gamma$ & $\begin{array}{c}L_{\mathrm{CPL} 10^{37}} \\
\mathrm{erg} \mathrm{s}^{-1}\end{array}$ \\
\hline 1 & $1.37 \pm 0.04$ & $7.1 \pm 0.5$ & $2.3 \pm 0.2$ & $2.20 \pm 0.04$ & 11.2 \\
2 & $1.37 \pm 0.03$ & $7.1 \pm 0.4$ & $2.3 \pm 0.2$ & $2.17 \pm 0.03$ & 11.4 \\
3 & $1.42 \pm 0.03$ & $6.7 \pm 0.4$ & $2.4 \pm 0.2$ & $2.13 \pm 0.03$ & 11.5 \\
4 & $1.50 \pm 0.03$ & $5.9 \pm 0.3$ & $2.3 \pm 0.1$ & $2.12 \pm 0.03$ & 11.9 \\
5 & $1.62 \pm 0.04$ & $5.1 \pm 0.3$ & $2.3 \pm 0.1$ & $2.09 \pm 0.03$ & 12.1 \\
6 & $1.71 \pm 0.05$ & $4.5 \pm 0.3$ & $2.2 \pm 0.1$ & $2.05 \pm 0.03$ & 12.3 \\
7 & $1.89 \pm 0.06$ & $3.8 \pm 0.3$ & $2.3 \pm 0.1$ & $2.04 \pm 0.04$ & 12.6 \\
8 & $2.02 \pm 0.06$ & $3.4 \pm 0.2$ & $2.6 \pm 0.2$ & $2.01 \pm 0.04$ & 12.5 \\
9 & $2.16 \pm 0.06$ & $3.1 \pm 0.2$ & $2.8 \pm 0.2$ & $2.01 \pm 0.15$ & 12.7 \\
10 & $2.18 \pm 0.06$ & $3.1 \pm 0.2$ & $2.8 \pm 0.2$ & $1.95 \pm 0.05$ & 12.8 \\
11 & $2.22 \pm 0.07$ & $3.1 \pm 0.2$ & $3.0 \pm 0.3$ & $1.94 \pm 0.06$ & 12.8 \\
12 & $2.25 \pm 0.10$ & $3.2 \pm 0.4$ & $3.4 \pm 0.3$ & $1.96 \pm 0.11$ & 13.0 \\
\hline
\end{tabular}

emission. The power law photon index $\Gamma$ decreased systematically by $15 \%$ during flaring as seen in Fig. 4 .

The iron line had a non-flaring $E W$ of $60 \pm 20 \mathrm{eV}$ in the 1997 data, and $90 \pm 30 \mathrm{eV}$ in the 1999 data. We do not show $E W$ in Tables 2 and 3 for the growth of flares, because the continuum flux is changing; the line intensity is more relevant. In the 1997 data, the intensity of the $6.4 \mathrm{keV}$ line did not change, and it will be relevant that $L_{\mathrm{BB}}$ was also constant. In the 1999 data, the line intensity $I_{\text {line }}$ did change and is plotted against $L_{\mathrm{BB}}$ and $L_{\mathrm{CPL}}$ (the luminosity of the Comptonized emission) in Fig. 5. This shows that during the initial growth of flaring the line intensity does not change significantly although $L_{\mathrm{CPL}}$ is increasing; $L_{\mathrm{BB}}$ is constant at this stage. However, in the second stage of flare growth, $L_{\mathrm{BB}}$ increases (Fig. 4), and then the line intensity increases. In Fig. 5, the best-fit line to the variation of $I_{\text {line }}$ with $L_{\mathrm{BB}}$ is shown, this taking into account the $90 \%$ confidence errors in both $y$ and $x$. This line has gradient $8.57 \pm 0.69 \times 10^{-5}$ photon $\mathrm{cm}^{-2} \mathrm{erg}^{-1}$, showing that the line intensity is correlated with the blackbody luminosity with high significance. This is the first time that a line variation has been linked to the variation of a particular continuum emission component; this will be discussed further in Sect. 4.
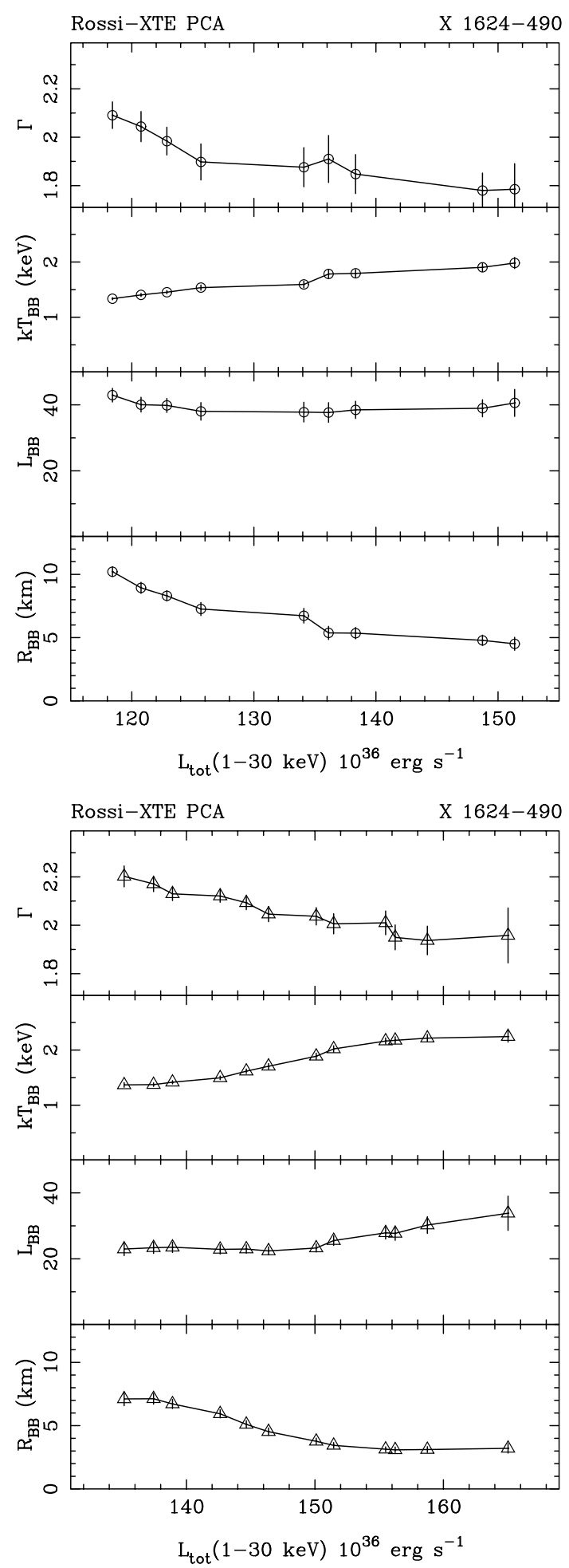

Fig. 4. Upper: spectral fitting results from the 1997 observation: blackbody parameters and power law photon index $\Gamma$. The blackbody luminosity is in units of $10^{36} \mathrm{erg} \mathrm{s}^{-1}$. Lower: Corresponding results from the 1999 observation.

The data of Fig. 4 are replotted in Fig. 6 as $k T_{\mathrm{BB}}$ against $L_{\text {Tot }}$ for both $R X T E$ observations, plus a point from the BeppoSAX observation (Bałucińska-Church et al. 2000), when the source was substantially less bright. We have made comparisons of $R X T E$ PCA Crab data in the 

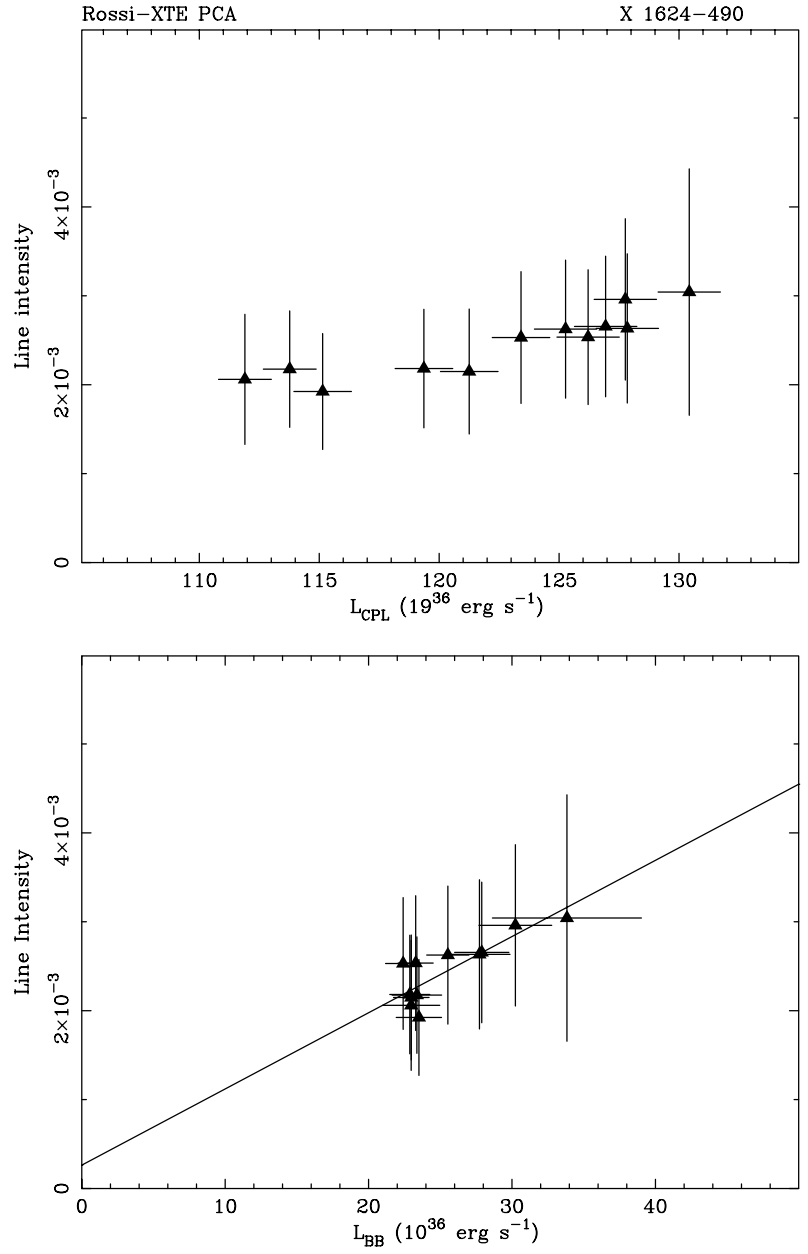

Fig. 5. Variation of iron line intensity (in photon $\mathrm{cm}^{-2} \mathrm{~s}^{-1}$ ) with the luminosity of the Comptonized emission (upper panel), and with the blackbody luminosity (lower panel). The best-fit line is shown in the lower panel (see text).

band 3-20 keV (obtained before and after the 1999 observation of X 1624-490) with BeppoSAX MECS data of 1998, October, which shows that systematic differences between flux from the two instruments amount to no more than $17 \%$, (higher in $R X T E$ ). Figure 6 shows that at the peak of flaring in the 1999 data, $k T_{\mathrm{BB}}$ saturates at a constant value at the same time that $R_{\mathrm{BB}}$ becomes constant at a low value (Fig. 4). During this stage of flaring, $L_{\mathrm{BB}}$ increases, eventually becoming fixed when the emitting area and $k T_{\mathrm{BB}}$ become constant. In Fig. $7, L_{\mathrm{BB}}$ is plotted against blackbody temperature $k T_{\mathrm{BB}}$. The approximate constancy of $L_{\mathrm{BB}}$ in the early stages of flaring is apparent. Also shown is a line drawn through the penultimate point of the 1999 observation before the peak of flaring, representing the relation $L_{\mathrm{BB}}=A \sigma T^{4}$. In this second stage of flaring as $R_{\mathrm{BB}}$ becomes constant, further increase in $L_{\mathrm{BB}}$ is due to temperature increase at $\sim$ constant area, so that the data obey Stefan's Law.

Next, the results are re-expressed in the way used by Church \& Bałucińska-Church (2001) in a survey of LMXB sources using $A S C A$ and BeppoSAX. In Fig. 8 we show the variation of the half-height $h$ of the emission region on

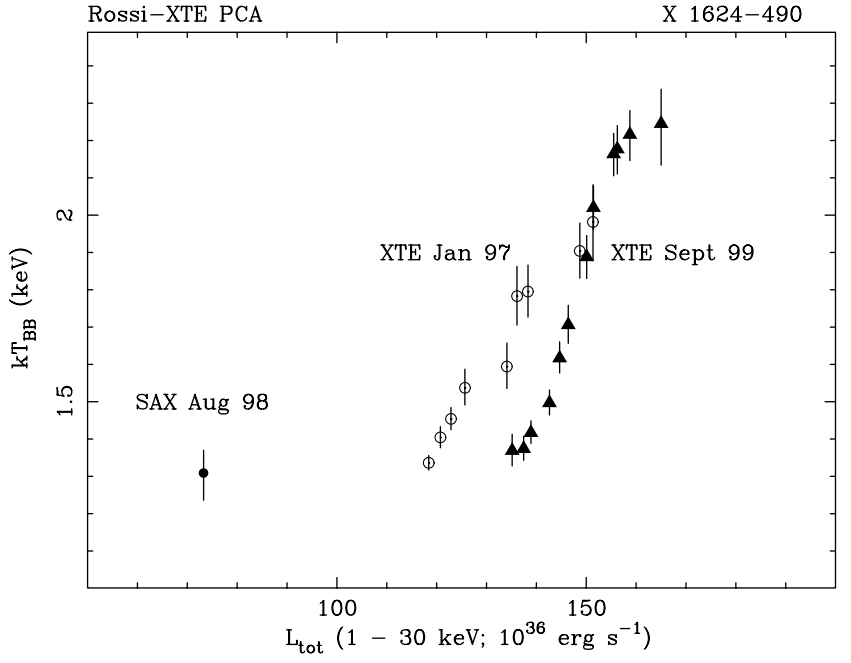

Fig. 6. Variation of blackbody temperature $k T_{\mathrm{BB}}$ with source luminosity for the 1997 and 1999 RXTE observations and the 1998 BeppoSAX observation.

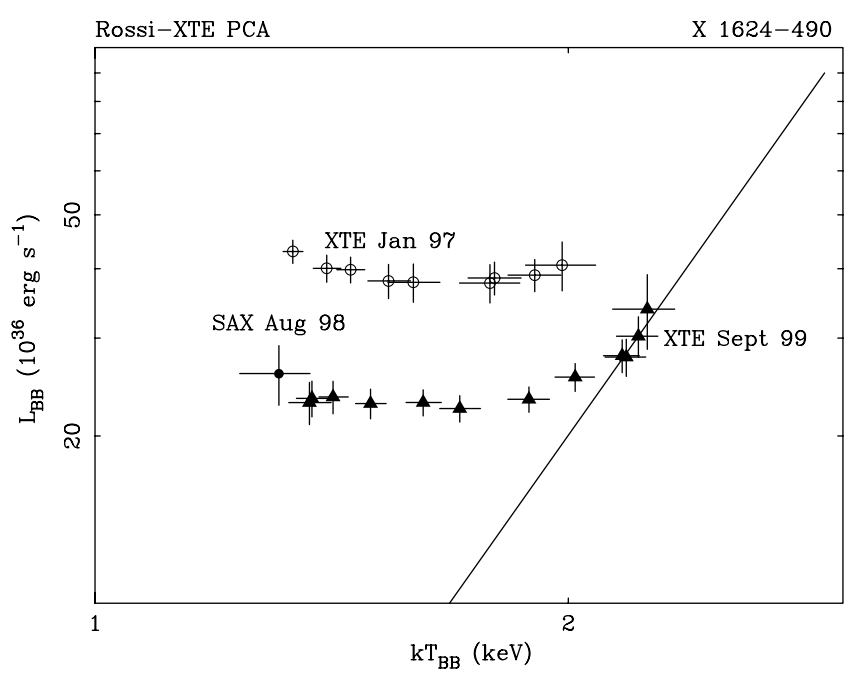

Fig. 7. Blackbody luminosity as a function of blackbody temperature which increases as flares evolve. The constancy of $L_{\mathrm{BB}}$ over most of the flaring can be seen, and the final increase of luminosity agreeing with a $T^{4}$ law, as the blackbody emitting area becomes constant (see text).

the neutron star as a function of $L_{\text {Tot }}$. It is assumed that emission is from an equatorial strip on the star with area $4 \pi h R$ (a sphere intersected by two parallel planes) equal to $4 \pi R_{\mathrm{BB}}^{2}$. Thus, $h=R_{\mathrm{BB}}^{2} / R$, and it is assumed that $R$ is $10 \mathrm{~km}$. Also shown are calculated values of the equilibrium half-height $H_{\mathrm{eq}}$ of the inner radiatively-supported accretion disk given approximately by the equation (Frank et al. 1992):

$H=\frac{3 \sigma \dot{M}}{8 \pi m_{\mathrm{p}} c}\left[1-\left(\frac{R}{r}\right)^{1 / 2}\right]=H_{\mathrm{eq}}\left[1-\left(\frac{R}{r}\right)^{1 / 2}\right]$.

This equation shows that the disk height increases rapidly with radial distance from zero at the surface of the neutron star towards the equilibrium value $H_{\text {eq }}$. Most of the 


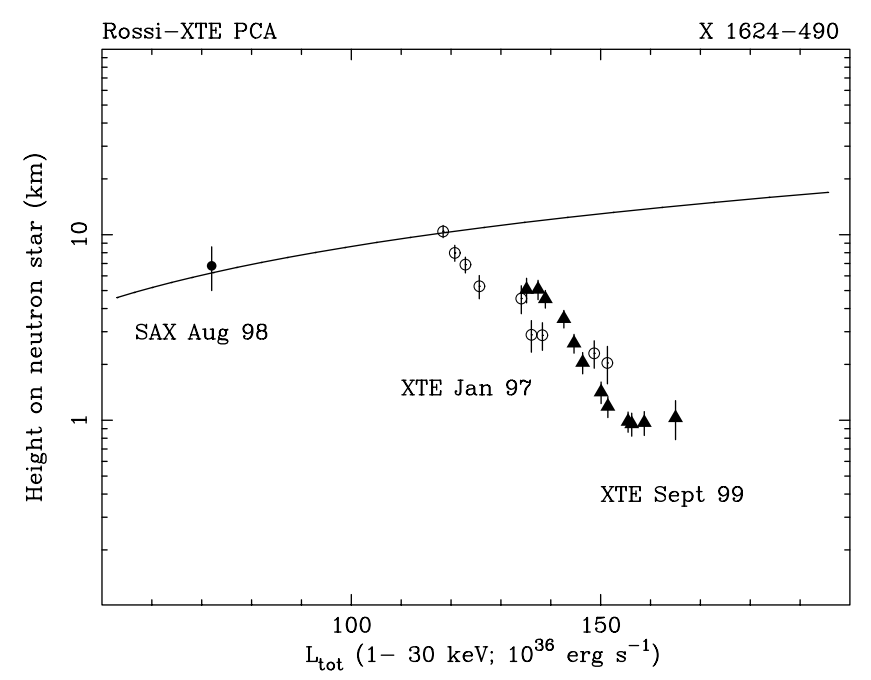

Fig. 8. Evolution of the blackbody emitter during flaring in X 1624-490; the curve shows the equilibrium height of the radiatively-supported inner disk $H_{\text {eq }}$.

increase takes place between $r=10$ and $r=20 \mathrm{~km}$, i.e. within $10 \mathrm{~km}$ of the stellar surface. In weak sources, with $L \sim 5 \times 10^{36} \mathrm{erg} \mathrm{s}^{-1}$, radiative support is over a limited radial extent so that $H_{\mathrm{eq}}$ is never achieved. However, in bright sources with $L \sim 10^{38} \mathrm{erg} \mathrm{s}^{-1}$, such as X 1624-490, the equilibrium height is achieved, and the radiativelysupported disk extends to a radial distance of $\sim 400 \mathrm{~km}$. For the sources in the LMXB survey there was found to be an agreement $h=H$ spanning 3 orders of magnitude in luminosity.

In the case of X 1624-490, we calculated $H_{\mathrm{eq}}$ from $L_{\mathrm{Tot}}$ using the expression $L_{\mathrm{Tot}}=G M \dot{M} / R$, where $M$ is the mass of the neutron star taken to be $1.4 M_{\odot}$. However the increase of luminosity in flaring probably does not mean an increase in $\dot{M}$ (as in X-ray bursts). Thus the curve of $H_{\mathrm{eq}}$ shown in Fig. 8 is appropriate for comparison of the non-flaring source in the 3 observations, but should not be taken to imply $\dot{M}$ increases in flares. It can be seen that there is good agreement between $h$ and $H_{\text {eq }}$ for the BeppoSAX and 1997 RXTE observations. In the 1997 data, $h=10 \mathrm{~km}$, i.e. the whole neutron star is emitting. During flaring, there is a strong depression of $h$ below $H_{\text {eq }}$ by a factor of $\sim 5$ at the peak of flaring. In the 1999 observation, it can be seen that the non-flaring source has $h$ already depressed below $H_{\text {eq }}$.

As flaring develops in the 1997 observation, $L_{\text {Tot }}$ increases from $1.15 \times 10^{38} \mathrm{erg} \mathrm{s}^{-1}$ to $1.5 \times 10^{38} \mathrm{erg} \mathrm{s}^{-1}$. The 1999 RXTE data shows similar behaviour, except that the non-flaring luminosity was higher at $1.3 \times 10^{38} \mathrm{erg} \mathrm{s}^{-1}$ rising to $1.7 \times 10^{38} \mathrm{erg} \mathrm{s}^{-1}$ in flares. It is important to decide whether the initial depression of $h$ below $H_{\text {eq }}$ is real so the effects of uncertainty in the Comptonization cutoff energy discussed previously were tested. The value of $E_{\mathrm{CO}}$ was allowed to vary between its smallest value $9 \mathrm{keV}$ obtained in free fitting and the maximum value $21 \mathrm{keV}$. The effect of this was that the total luminosity $(1-30 \mathrm{keV})$ varied as might be expected, from the best-fit value of $1.3 \times 10^{38} \mathrm{erg} \mathrm{s}^{-1}$ for $E_{\mathrm{CO}}=11.8 \mathrm{keV}$ by $\pm 0.1 \times 10^{38} \mathrm{erg} \mathrm{s}^{-1}$ for the non-flaring spectrum. However, there was essentially no change in the height of the emitting region on the neutron star. We can conclude that the dramatic changes shown in Fig. 7 are not affected by this uncertainty.

\section{Discussion}

The main result of our analysis is a neutron star blackbody radius which decreases strongly as flares develop. We firstly consider whether this can be influenced by the fact that we fitted a simple blackbody when it is often assumed that X-ray burst spectra, particularly at the peaks of bursts can have a modified blackbody spectral form, due to the effects of electron scattering in the neutron star atmosphere. There were two types of evidence for a modification taking place: blackbody temperatures of $2.5-3.0 \mathrm{keV}$ implying super-Eddington luminosities in bursts, and an anti-correlation of blackbody radius $R_{\mathrm{BB}}$ with $k T_{\mathrm{BB}}$ in a particular source (below) when a simple blackbody was fitted. It should be mentioned at this stage that it would be unwise not to fit a simple blackbody model to the present data as the spectral form depends on one parameter only $k T_{\mathrm{BB}}$, allowing straightforward interpretation of the results. Particular modified blackbody models have a flux depending on $k T$ and electron density and so provide no information on the emitting area unless some assumption is made about the electron density.

In the study of X-ray bursts, it was first shown by Swank et al. (1977) that the burst spectra were well described by a simple blackbody. This was supported by the fact that during burst decay, the flux varied approximately as $T^{4}$ (Hoffman et al. 1977). By fitting a simple blackbody model, burst peak blackbody temperatures of $\sim 2 \mathrm{keV}$ are generally found; however higher temperatures of $\sim 3 \mathrm{keV}$ have been measured, also obtained using high quality data from the Rossi-XTE PCA (Swank priv. comm.), so that high temperatures at the peak of some bursts cannot be doubted. As these high temperatures imply super-Eddington luminosities, theory was developed in which electron scattering dominated over free-free absorption in the opacity of the neutron star atmosphere during bursts, and the surface value of the radiation source function departed from Planck form and had a modified blackbody form. Modified blackbody spectral forms have been given for a constant density atmosphere which we will designate MBB1 (van Paradijs 1984) and for an exponential atmosphere MBB2 (Zel'dovich \& Shakura 1969). Modelling of the neutron star atmosphere has been carried out by Fujimoto et al. (1981) who derived conditions for stable and unstable hydrogen and helium burning, by Paczyński (1983), by London et al. (1984, 1986), Ebisuzaki et al. (1984), and Ebisuzaki (1987). London et al. modelled the atmosphere numerically allowing for the effects of Comptonization. Although the simpler models MBB1 and MBB2 have a spectral form which differs from the 


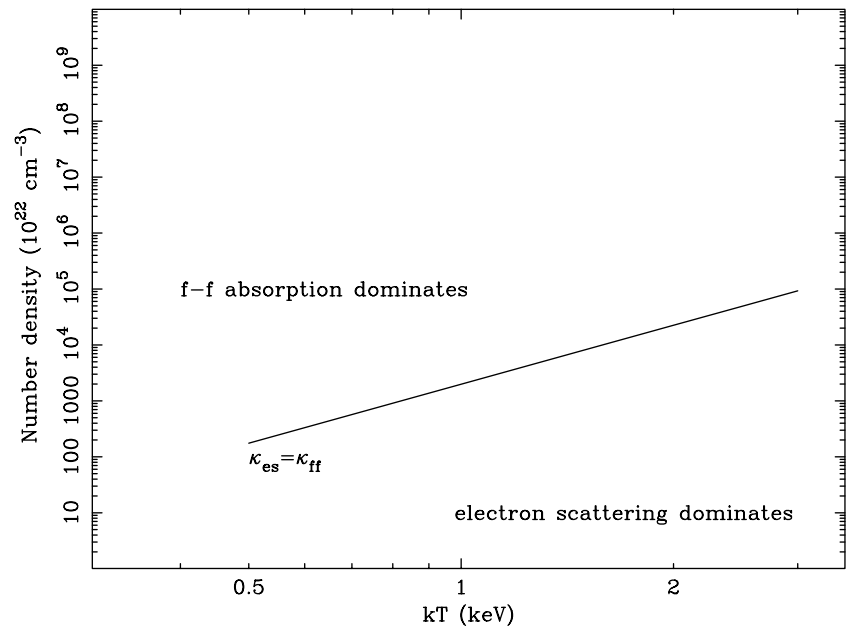

Fig. 9. Relative contributions of absorption and electron scattering to the opacity in the neutron star atmosphere as a function of number density and temperature $k T$.

Planck form, and could, in principle, be detected if sufficient count statistics were available, the more sophisticated numerical models have forms which are similar to the Planck form although the peak energy has a different relation to $k T$. However, when close to the Eddington limit, a substantial low-energy hump is expected (Madej 1991). In our work on LMXB, we have never found any indication of a departure from simple blackbody spectral shape in the quiescent spectra, all sources studied being well-fitted by using a simple blackbody plus cut-off power law model (e.g. Church \& Bałucińska-Church 1995; Bałucińska-Church et al. 1999; Smale et al. 2001). X-ray burst spectra have been well-fitted by a simple blackbody, e.g. using the Rossi-XTE PCA with its high sensitivity, it has been possible to examine burst spectra accumulated over times as short as $0.1 \mathrm{~s}$, and these are well-fitted by a simple blackbody as in 4U1728-34 (Swank 2001). One possibility remaining is that bursts with $k T \sim 3 \mathrm{keV}$ may have modified spectra, whereas those with $k T \sim 2 \mathrm{keV}$ do not.

Modification of the blackbody spectrum was expected on the grounds that in an X-ray burst, the density was "low" and the temperature "high", and we next address the question of how well the electron density is known. It is convenient to use a density-temperature diagram on which we can delineate the regions in which free-free and electron scattering processes dominate as shown in Fig. 9. Density can be expressed as electron number density, as mass density $\rho$, or as the vertically integrated mass column density $\Sigma$. The problem is that the value of density used in various theoretical treatments of X-ray bursting are very different. Fujimoto et al. (1981) provide values of $\Delta M \sim 10^{-12}-10^{-11} M_{\odot}$ for the total mass needed to be accumulated on the surface of the star for a nuclear flash to occur. From this, $\Sigma=10^{8}-10^{9} \mathrm{~g} \mathrm{~cm}^{-2}$ and if we assume the thickness of the atmosphere in the range $10-100 \mathrm{~cm}$, the average $\rho$ is $10^{6}-10^{8} \mathrm{~g} \mathrm{~cm}^{-3}$. However, the density can vary considerably from the mean, and be

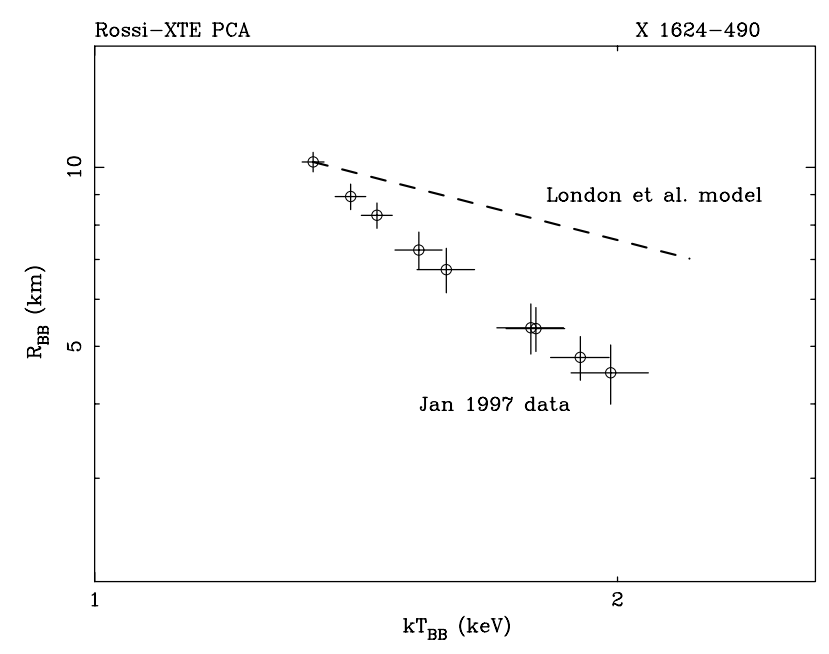

Fig. 10. Test of the hypothesis that the apparent decrease in blackbody area in flaring is not real by comparing the 1997 results with the $R-T$ relation predicted by the London et al. model atmosphere (dashed line) assuming that the emitting area remains constant (see text).

different in the hydrogen and helium burning shells (Joss \& Rappaport 1984). London et al. (1984) assume a value of $\Sigma=100 \mathrm{~g} \mathrm{~cm}^{-2}$. Ebisuzaki (1984) gives a range of mass densities in the surface layer for a burst luminosity of $20 \%$ of Eddington from $1-10^{4} \mathrm{~g} \mathrm{~cm}^{-3}$, which correspond to $\Sigma$ in the range $5-5 \times 10^{4} \mathrm{~g} \mathrm{~cm}^{-2}$. Paczyński (1983) used values of surface mass $(\Delta M)$ similar to those of Fujimoto et al. (1981). Thus these values correspond to $\Sigma$ spanning the range $5-10^{9} \mathrm{~g} \mathrm{~cm}^{-2}$ and to electron number densities $n_{\mathrm{e}}$ in the range $10^{24}-10^{32} \mathrm{~cm}^{-3}$. While an atmosphere with $n_{\mathrm{e}}=10^{24} \mathrm{~cm}^{-3}$ is clearly dominated by electron scattering, this is not the case for higher values of electron density. The calculation of densities in neutron star atmospheres during bursts is complex and it does not appear possible yet to be definite about values.

The second piece of evidence for modification of the blackbody in X-ray bursts was the apparent decrease in blackbody radius with increasing temperature $k T$ when a simple blackbody model was fitted to Tenma and Exosat data of bursts in XB 1636-536 (Inoue et al. 1984; Sztajno et al. 1985). In these data, $R_{\mathrm{BB}}$ decreased by about $40 \%$ as $k T_{\mathrm{BB}}$ increased from 1 to $2 \mathrm{keV}$. Sztajno et al. showed that the apparent decrease was consistent with a constant emitting area if the model atmospheres of London et al. $(1984,1986)$ were used. We will make the same comparison with the present data. London et al. construct models for various values of the emitted flux $f$, which they re-express in terms of an effective temperature $T_{\text {eff }}$, defined via $f \sim T_{\text {eff }}^{4}$. Fitting a blackbody to the emission of their model atmospheres provides a spectral or colour temperature $T_{\text {spec }}$, so that if the luminosities of the fitted blackbody and generated spectrum are equal:

$4 \pi R_{\mathrm{BB}}^{2} \sigma T_{\mathrm{spec}}^{4}=$ const $\cdot R^{2} T_{\mathrm{eff}}^{4}$.

Assuming $R$ is constant gives $R_{\mathrm{BB}} \propto\left(T_{\text {eff }} / T_{\text {spec }}\right)^{2}$. Using the spectral hardening factor $T_{\mathrm{spec}} / T_{\mathrm{eff}} \sim T_{\mathrm{eff}}^{3 / 5}$ of 
Table 4. Results of fitting non-flare emission by simple blackbody and flaring by a modified blackbody spectral form.

\begin{tabular}{llrr}
\hline & Non-flare & Mid-flare & Peak-flare \\
\hline & Simple BB & Modified BB & Modified BB \\
$k T(\mathrm{keV})$ & $1.37 \pm 0.04$ & $2.30 \pm 0.07$ & $3.46 \pm 0.10$ \\
norm & $1.02 \pm 0.09$ & $1.86 \pm 0.11$ & $3.35 \pm 0.53$ \\
$R(\mathrm{~km})$ & $7.1 \pm 0.5$ & 7.1 & 7.1 \\
$n_{\mathrm{e}}\left(\mathrm{cm}^{-3}\right)$ & $\cdots$ & $3.7 \pm 0.7 \times 10^{23}$ & $1.9 \pm 0.3 \times 10^{23}$ \\
\hline
\end{tabular}

Norm has units of $10^{37} \mathrm{erg} \mathrm{s}^{-1}$ at $10 \mathrm{kpc}$.

London et al., we obtain $R_{\mathrm{BB}} \sim 1 / T_{\text {spec }}^{3 / 4}$ for the expected dependence of the apparent decrease in blackbody radius with increasing measured burst temperature. In the case of the present flare data if we assume some modification of the spectrum takes place, as $k T$ increases from 1.34 to $1.98 \mathrm{keV}$ (1997 data), we would expect a decrease in $R_{\mathrm{BB}}$ of $34 \%$, whereas the actual increase is by a factor of 2.3, and these changes are compared in Fig. 10. Thus, it does not seem very likely on the basis of the London et al. model that the emitting area can actually remain constant while appearing to decrease by this large factor.

If we use the simpler MBB1 modified blackbody model, the luminosity is related to the electron density via const. $\cdot R^{2}(k T)^{9 / 4} n_{\mathrm{e}}^{1 / 2}$ (with a similar relation for MBB2). To demonstrate the constraints imposed on $n_{\mathrm{e}}$, we fitted the 1999 data using the model MBB1 + CPL. This fitting provided good values of $\chi^{2} /$ d.o.f.; results are shown in Table 4 . We assume that the spectrum of the non-flaring source is an unmodified blackbody, but that mid-flare and peak-flare can be modified. In the non-flare emission, the blackbody radius $R_{\mathrm{BB}}$ was $7.1 \mathrm{~km}$. Assuming that this does not change in flaring, we use the measured MBB1 normalization and $k T$ values to derive the electron density at the peak of flaring, $n_{\mathrm{e}}=1.9 \times 10^{23} \mathrm{~cm}^{-3}$. Not only is this fine-tuning of electron density values, but the value is relatively low in terms of expected values in model atmospheres as discussed above. Moreover, the electron density would have to decrease by a factor of two between midflaring and the peak of flaring to keep $R$ constant. It is unlikely that $n_{\mathrm{e}}$ decreases in flaring and so for this simple model also, it is unlikely that the emitting area does not decrease.

However, it is not proven that a modification does take place in flaring, and there are arguments that it does not. Firstly, the value of $k T \sim 2 \mathrm{keV}$ obtained at the peaks of flares does not demand a modification of the spectrum in the way that super-Eddington values of $k T=3 \mathrm{keV}$ do in X-ray bursts. Secondly, X-ray flaring is much less well understood than X-ray bursting and so our knowledge of densities in the neutron star atmosphere is even more uncertain as no theoretical model of the atmosphere during flaring exists at present. We have previously suggested (Sect. 3.1) that an X-ray flare consists of a superposition of X-ray bursts. The recurrence time between these

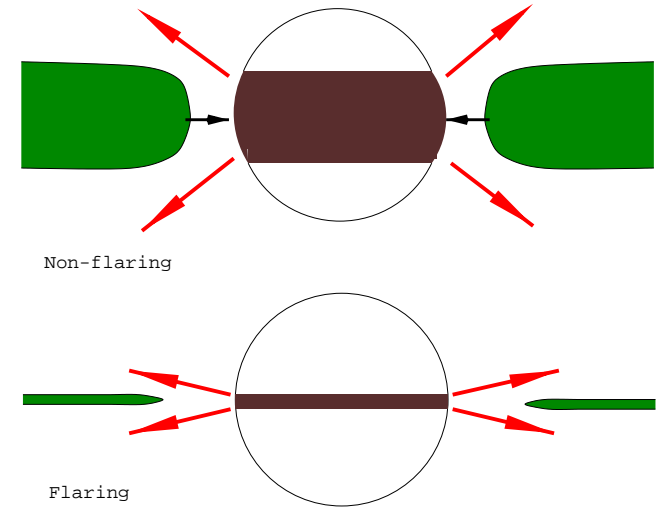

Fig. 11. Schematic diagram showing the result from spectral fitting that the emitting area of the neutron star blackbody decreases strongly during flaring. The top panel shows the calculated height $H$ of the radiatively-supported inner disk which agrees well with the neutron star emitter height $h$ in the nonflaring state. One possibility (see text) is that the vertical extent of the inner disk is decreased in flaring (lower panel).

is expected to be short as observed, because of the much higher values of mass accretion rate compared with a burst source. The 1-30 keV luminosity of X 1624-490 in the observations discussed here is $\sim 10^{38} \mathrm{erg} \mathrm{s}^{-1}$, i.e. considerably higher than that of a typical burst source. Consequently it is likely that the densities in the atmosphere are more than 10 times higher making it less likely that electron scattering will dominate the opacity. In summary, there is no observational evidence of a modification of the blackbody spectrum. However, if a modification did take place, it is unlikely that this can explain the large observed decrease in area, and we conclude that the main results of the analysis made using a simple blackbody are correct.

We now discuss possible reasons for the decrease in emitting area. Results of the $A S C A$ survey of LMXB (Church \& Bałucińska-Church 2001) showed that the level of neutron star blackbody emission can be explained by assuming that the half-height of emitter on the star $h$ is equal to the half-height $H$ of the inner radiativelysupported disk (see Sect. 1). Two possible explanations exist (see Sect. 1): either there is radial flow across the gap between disk and star (mapping the value of $H$ onto $h$ ), or $H$ is simply a measure of $\dot{M}$, and the mechanism of Inogamov \& Sunyaev (1999) (accretion flow spreading on the stellar surface) determines $h$. If the first of these is correct, then radiation pressure can affect the inner disk by material being removed at the upper and lower surfaces by radiation pressure, leading directly to a decrease in $h$ and of $\dot{M}$ reaching the star in flaring, as shown in Fig. 11. If the second mechanism is correct, then we need the accretion flow between disk and star to be impeded by radiation pressure. In either case, it is important to compare the levels of blackbody emission obtained from spectral fitting with the Eddington limit to test whether the effects of radiation pressure should be expected. If the emitting area is reduced at the peak of flaring to a narrow equatorial strip, radiation will not be isotropic. For an 
observer at angle $\theta$ from the equatorial plane, the observed flux will be $f_{\text {obs }}=f \cdot \cos \theta$ where $f$ is the flux in the plane. For X 1624-490, $\theta \sim 30^{\circ}$. To test whether Eddington limiting takes place in the equatorial plane, we should compare $f=f_{\text {obs }} / \cos \theta$ with $f_{\text {Edd }}$.

Taking into account the non-Newtonian gravitation near the neutron star and possible variations in the composition of neutron star atmosphere so that the opacity may not equal the Thompson scattering opacity, there will be a local value of critical luminosity $L_{\text {crit }}=$ $L_{\mathrm{Edd}} \cdot \frac{\kappa}{\kappa_{\mathrm{T}}} \cdot(1+z)$ where $L_{\mathrm{Edd}}$ is the normal Eddington limiting luminosity, $\kappa_{\mathrm{T}}$ is the Thomson scattering opacity, $\kappa$ is the actual opacity and $z$ the gravitational redshift (Paczyński 1983). For a distant observer, this critical value will be reduced by the gravitational redshift to $L_{\mathrm{crit}, \infty}$, so that

$L_{\text {crit }, \infty}=\frac{4 \pi G M c}{\kappa_{\mathrm{T}}} \cdot \frac{\kappa_{\mathrm{T}}}{\kappa} \cdot \frac{1}{(1+z)}$

where $4 \pi G M c / \kappa_{\mathrm{T}}$ is the standard Eddington limiting luminosity $L_{\text {Edd }}=1.76 \times 10^{38} \mathrm{erg} \mathrm{s}^{-1}$ for a $1.4 M_{\odot}$ neutron star. This depends on composition since $\kappa_{\mathrm{T}} \simeq 0.2(1+X)$ where $X$ is the fraction by weight of hydrogen. The factor $1 /(1+z)=\sqrt{1-2 G M / R c^{2}}$ in terms of the mass $M$ and radius $R$ of the star. We will compare our measured blackbody luminosities with the Eddington limit

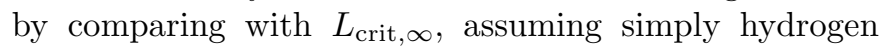
composition and a value of $z=0.34$ appropriate to the surface at $R=10 \mathrm{~km}$ of a $1.4 M_{\odot}$ neutron star. Thus $L_{\text {crit }, \infty}=1.31 \times 10^{38} \mathrm{erg} \mathrm{s}^{-1}$.

In both the 1997 and 1999 data, the observed blackbody luminosity $L_{\mathrm{BB}}$ remains less than $4 \times 10^{37} \mathrm{erg} \mathrm{s}^{-1}$. Thus the flux in the equatorial plane corresponds to a luminosity of $\sim 5 \times 10^{37} \mathrm{erg} \mathrm{s}^{-1}$, i.e. about a factor of 2.5 less than $L_{\text {crit, } \infty}$. This calculation suggests that the effects of radiation pressure are important. In the 1997 data, $L_{\mathrm{BB}}$ remains about constant at $4 \times 10^{37} \mathrm{erg} \mathrm{s}^{-1}$ and the blackbody radius $R_{\mathrm{BB}}$ decreases from its non-flaring value of $10.2 \pm 0.4 \mathrm{~km}$ as $k T_{\mathrm{BB}}$ increases. In the 1999 data, $R_{\mathrm{BB}}$ starts at a somewhat lower level than in 1997 at $7.1 \mathrm{~km}$ so that the effects seen in flaring are already taking place in the non-flaring emission. The effects of radiation pressure clearly extend over a range of blackbody luminosity values, but at the peak of flaring further increase of $L_{\mathrm{BB}}$ is prevented due to both $k T_{\mathrm{BB}}$ and $R_{\mathrm{BB}}$ reaching stable values. Exactly how the lower limit of $R_{\mathrm{BB}}$ is caused is not clear. The processes discussed of increasing $k T_{\mathrm{BB}}$ and decreasing $R_{\mathrm{BB}}$ constitute a mechanism for limiting the blackbody luminosity and thus an Eddington limiting effect not previously known. At the peak of flaring, the mass accretion flow to the star will be reduced below the nonflaring case by the changes in the inner disk and star, the radiation pressure will fall, and the height of the emitting region on the star increase. Thus, the flare is self-limiting and the system will return to the non-flare state on a similar timescale to that of flare growth.

We next discuss further the implications of the relation $h=H$ obtained from the $A S C A$ and BeppoSAX survey (Church \& Bałucińska-Church 2001), and supported by the present work and the two possible explanations referred to previously. The first explanation involves radial flow between inner disk and star. Although there has been a large amount of theoretical work on advective flow in black hole binaries, e.g. Abramowicz et al. (1996), and references therein, and on the nature of the disk itself, such as on slim disks (Abramowicz et al. 1988; Igumenschev et al. 1998), there has been relatively little corresponding work on the accretion flow between the inner disk and neutron star in LMXB. It is not known whether the sonic point lies within the star or within the inner disk. However, Popham \& Sunyaev (2001) investigate the nature of the inner accretion disk around a neutron star, assuming a boundary layer within the disk, and show that the radial velocity increases by about two orders of magnitude leading to advective flow within the disk and increase in disk height due to increased heating. This does not answer the question, of course, as to whether material can flow across the gap between the inner disk edge and the star, although Popham \& Sunyaev comment that they would intuitively expect material to fall onto the star in a wide belt. Two- or three-dimensional modelling may reveal whether such radial flows are possible. It has been suggested that the model of radial flow between an inner radiative disk and the star is invalid as the inner disk may be unstable to the radiation pressure instability discussed by Janiuk et al. (2000) for a $10 M_{\odot}$ black hole. However, scaling from a $10 M_{\odot}$ black hole to a $1.4 M_{\odot}$ system indicates an instability timescale of $\sim 30 \mathrm{~s}$, and this, even if present, may not disrupt the disk.

The alternative explanation of our results involves the flow of accretion material on the surface of the neutron star as proposed by Inogamov \& Sunyaev (1999). The ageement between $h$ and $H$ would result from $H$ being a measure of the total luminosity, not from $H$ directly determining $h$ in some way. However, preliminary results of a comparison of this model with the survey results show that the observed blackbody luminosities are $\sim 3$ times larger than predicted by the theory (Church et al. 2001). This implies therefore, that radial flow may be the dominant factor determining the neutron star blackbody emission. The present results on flaring suggest for the radial flow explanation that radiation pressure can reduce the inner disk height so that both the emitting area on the star, and the mass accretion rate reaching the star are reduced. Possibly, the accretion flow could be affected without the disk height changing. In the case of the Inogamov \& Sunyaev mechanism, the decrease of $\dot{M}$ would lead to a decrease of emitting area.

The present results are difficult to reconcile with the possibility that the blackbody emission in LMXB could originate in the accretion disk as multi-colour disk (MCD) blackbody emission. There would be no reason to expect any agreement between $H$ and $h$, as $h$ would be related to the inner radius $r_{\mathrm{i}}$ of the disk blackbody (via $h=r_{\mathrm{i}}^{2} / R$ ). The survey of LMXB (Church \& Bałucińska-Church 2001) showed that for the majority of sources, a Comptonization 
plus MCD blackbody model required unphysical values of inner disk radius, substantially less than the radius of the neutron star. The absence of disk blackbody emission in LMXB can however, be understood in terms of the very extended flat, nature of the ADC revealed by dip ingress time measurements (Sect. 1). An extended ADC of radius typically $50000 \mathrm{~km}$ and large optical depth (Church 2001) will completely cover the hot X-ray emitting inner accretion disk, so that all thermal emission will be Comptonized and no disk blackbody component seen.

Finally, we will discuss the broad iron line at $\sim 6.4 \mathrm{keV}$. In Paper I, we reported the detection of this line in nondipping, non-flaring emission. It has an equivalent width of $\sim 60 \mathrm{eV}$ and $\sim 90 \mathrm{eV}$ for the 1997 and 1999 observations, respectively. Asai et al. (2000) made the first detection of a broad line in this source at $6.4_{-0.3}^{+0.1} \mathrm{keV}$ using $A S C A$ data; with an $E W$ of $13_{-9}^{+13} \mathrm{eV}$. In the present paper, we show that the line intensity is proportional to the luminosity of the blackbody component, the first demonstration that a line intensity is linked to a particular component of the continuum. Iron line emission is not well understood in LMXB with conflicting models for line origin. Asai et al. (2000) from their extensive study of iron lines in LMXB with $A S C A$ find that the line energy had a mean value of $6.57 \mathrm{keV}$ in 20 sources and argue that lines are formed by radiative recombination of photoionized plasma in the ADC, also proposed by Hirano et al. (1987) and White et al. (1985, 1986). In many sources, the line energy is $\sim 6.7 \mathrm{keV}$ strongly suggesting a blend of Fe XXV lines at $6.63,6.67$ and $6.70 \mathrm{keV}$ formed by recombination. Although there is some scatter in the line energies between extremes of 6.2 and $7.0 \mathrm{keV}$ it is thought likely that the lines in all sources have a common origin. In general, measurement of line energies of $6.4-6.5 \mathrm{keV}$ implying fluorescence has been taken to indicate origin in the accretion disk (e.g. Barret et al. 2000), although the number of detections of iron lines at these energies in LMXB is small. In the case of X 1624-490, the variation of the line in dipping (Paper I) is well described by giving the line the same covering factor as the ADC which supports the origin of the line in the ADC although the measured energy is unusually low at $6.4 \mathrm{keV}$. For the line energy to peak at $6.4 \mathrm{keV}$, an ionization parameter $\xi=L / n r^{2} \sim 100$ is required which may be possible if the density in the ADC is high. However, it is difficult to reconcile this line with the theoretical expectation that the electron temperature in the $\mathrm{ADC}$ of several $\mathrm{keV}$ might be expected to prevent formation of a $6.4 \mathrm{keV}$ line.

An iron line produced in the ADC will have no bearing on the non-detection of a reflection component in this source. In fact, there has been a general lack of detections of reflection components in LMXB and reported detections in a small number of sources have been ambiguous. Barret et al. (2000) presented evidence for a weak reflection component in GS 1826-238 and SLX 1735-269. However, in the first case, the spectrum was modelled with a one-component Comptonization term only but without a thermal component, which our previous work, e.g. the $A S C A$ survey of LMXB (Church \& Bałucińska-Church 2001) indicates is always present and would be detectable at the measured luminosity of the source. In the second case, the quality of fit was acceptable $\left(\chi^{2} /\right.$ d.o.f. $\left.=45 / 59\right)$ before a reflection component was added. In a Ginga observation of XB 1608-522, broad residuals above $7 \mathrm{keV}$ could be modelled either by partial absorption or a reflection component (Yoshida et al. 1993). In all of our previous work using the $\mathrm{BB}+\mathrm{CPL}$ model, there has been no trace of broad residuals indicating the presence of an additional continuum component. We can suggest a possible reason for the lack of detections. We have demonstrated the very extended, flat nature of the ADC (Church 2001; Smale et al. 2001), with radius $r_{\mathrm{ADC}}$ typically $50000 \mathrm{~km}$, or $15 \%$ of the disk radius, and particularly large in X 1624-490 (see Sect. 1). Thus the ADC will cover all of the inner disk and act as a "hot" reflector preventing illumination of the disk by the neutron star so preventing reflection. In addition, the large values of optical depth obtained for the ADC (Church 2001) will mean that any reflection component due to illumination by the ADC itself will not penetrate the ADC to the observer. In $\mathrm{Cyg} \mathrm{X}-1, r_{\mathrm{ADC}}$ is much smaller (Church 2001) and there is evidence for a reflection component, suggesting that LMXB neutron star binaries may differ from Black Hole binaries in this respect due to the strong effects of the neutron star in forming an extended ADC.

Future work will concentrate on further investigation of flaring in sources such X 1624-490 and on the flaring branch of Z-track sources. It is clear that spectral evolution during flaring can provide valuable information on the inner disk/neutron star interface not otherwise available.

\section{References}

Abramowicz, M. A., Czerny, B., Lasota, J.-P., \& Szuszkiewicz, E. 1988, ApJ, 332, 646

Abramowicz, M. A., Chen, X.-M., Granath, M., \& Lasota, J.-P. 1996, ApJ, 471, 762

Angelini, L., Parmar, A. N., \& White, N. E. 1997, Proc. IAU Colloq. 163, ed. D. T. Wickramasinghe, G. V. Bicknell, \& L. Ferrario (Port Douglas, 1996)

Asai, K., Dotani, T., Nagase, F., \& Mitsuda, K. 2000, ApJS, 131,571

Bałucińska-Church, M., Church, M. J., Oosterbroek, T., et al. 1999, A\&A, 349, 495

Bałucińska-Church, M., Humphrey, P. J., Church, M. J., \& Parmar, A. N. 2000, A\&A, 360, 583

Barret, D., Olive, J. F., Boirin, L., et al. 2000, ApJ, 533, 329

Canizares, C. R., Clark, G. W., Li, F. K., et al. 1975, ApJ, 197,457

Church, M. J. 2001, Proceedings of 33rd Scientific Assembly of COSPAR, Warsaw, July 2000, Adv., Space Res. in press

Church, M. J., \& Bałucińska-Church, M. 2001, A\&A, 369, 915

Church, M. J., \& Bałucińska-Church, M. 1995, A\&A, 300, 441

Church, M. J., Inogamov, N. A., \& Bałucińska-Church, M. 2001, A\&A, in preparation

Church, M. J., Dotani, T., Bałucińska-Church, M., et al. 1997, ApJ, 491, 388 
Church, M. J., Bałucińska-Church, M., Dotani, T., \& Asai, K. 1998a, ApJ, 504, 516

Church, M. J., Parmar, A. N., Bałucińska-Church, M., et al. 1998b, A\&A, 338, 556

Czerny, B., \& Elvis, M. 1987, ApJ, 321, 305

Ebisuzaki, T. 1987, PASJ, 39, 287

Ebisuzaki, T., Hanawa, T., \& Sugimoto, D. 1984, PASJ, 36, 551

Fujimoto, M. Y., Hanawa, T., \& Miyaji, S. 1981, ApJ, 246, 267

Frank, J., King, A. R., \& Raine, D. 1992, Accretion Power in Astrophysics (Cambridge University Press)

Hasinger, G., Priehorsky, W. C., \& Middleditch, J. 1989, ApJ, 337,843

Hasinger, G., \& van der Klis, M. 1989, A\&A, 225, 79

Hirano, T., Hayakawa, S., Nagase, F., Masai, K., \& Mitsuda, K. 1987, PASJ, 39, 619

Hoffman, J. A., Lewin, W. H. G., \& Doty, J. 1977, ApJ, 217, L23

Igumenschev, I. V., Abramowicz, M. A., \& Novikov, I. D. 1998, MNRAS, 298, 1069

Inogamov, N. A., \& Sunyaev, R. A. 1999, Astron. Lett., 25, 269

Inoue, H., Waki, I., Koyama, K., et al. 1984, PASJ, 36, 831

Janiuk, A., Czerny, B., \& Siemiginowska, A. 2000, ApJ, 542, L33

Jones, M. H., \& Watson, M. G. 1989, Proc. of 23rd ESLAB Symp., Bologna

Joss, P. C., \& Rappaport, S. A. 1984, ARA\&A, 22, 537

London, R. A., Taam, R. E., \& Howard, W. M. 1984, ApJ, 287, L27
London, R. A., Taam, R. E., \& Howard, W. M. 1986, ApJ, 306,170

Madej, J. 1991, ApJ, 376, 161

Paczyński, B. 1983, ApJ, 267, 315

Popham, R., \& Sunyaev, R. A. 2001, ApJ, 547, 355

Schulz, N. S., Hasinger, G., \& Trümper, J. 1989, A\&A, 225, 48

Shakura, N. I., \& Sunyaev, R. A. 1976, MNRAS, 175, 613

Smale, A. P., Church, M. J., \& Bałucińska-Church, M. 2001, ApJ, 550, 962

Swank, J. H. 2001, Proc. of Cosmic Explosions, Maryland, October 2000, ed. S. S. Holt, \& W. W. Zhang, AIP Conf. Proc., 522, in press

Swank, J. H., Becker, R. H., Boldt, E. A., et al. 1977, ApJ, 212,173

Sztajno, M., van Paradijs, J., Lewin, W. H. G., et al. 1985, ApJ, 299, 487

van Paradijs, J. 1984, Proc. of US-Japan Conf. on Galactic and Extragalactic X-ray Sources, Tokyo 1984, ed. Y. Tanaka

Watson, M. G., Willingale, R., King, A. R., Grindlay, J. E., \& Halpern, J. 1985, IAU Circ., 4051

White, N. E., \& Swank, J. H. 1982, ApJ, 253, L61

White, N. E., Peacock, A., Hasinger, G., et al. 1986, MNRAS 218,219

White, N. E., Peacock, A., \& Taylor, B. G. 1985, ApJ, 296, 475

Yoshida, K., Mitsuda, K., Ebisawa, K., et al. 1993, PASJ, 45, 605

Zel'dovich, Ya. B., \& Shakura, N. I. 1969, Astron. Zhur, 46, 225 